\title{
Gastrointestinale Wirkungen von Kümmelöl, Carvi aetheroleum
}

\author{
Tankred Wegener \\ Weinheim
}

\section{ZUSAMMENFASSUNG}

Das ätherische Kümmelöl aus Carum carvi L., Carvi aetheroleum, bestehend überwiegend aus den Terpenen (S)-(+)-Carvon und $(R)-(+)$-Limonen, wird bei Verdauungsbeschwerden, einhergehend mit Blähungen und Flatulenz, oder sogar bei flatulenzbedingten Koliken angewandt. Überwiegend aktuelle experimentelle Daten geben Hinweise oder bekräftigen die bereits früher postulierten Wirkungen. Zu nennen sind vor allem karminative (entblähende) Wirkungen im Gastrointestinum durch spasmolytische bzw. entkrampfende Eigenschaften, eine mögliche Hemmung der intestinalen Gärungsprozesse durch antimikrobielle (auf pathogene Keime beschränkt) Wirkungen, eine Volumenreduktion durch Hemmung der Schaumbildung von Magen- und Darmsaft und damit eine mögliche Reduktion blähungsbedingter Schmerzen sowie direkte antinozizeptive Eigenschaften. Ergebnisse aus klinischen Untersuchungen und Studien können die Wirkungen z. T. für das Kümmelöl alleine, besser für eine langjährig bewährte Kombination zusammen mit Pfefferminzöl, z. B. in der Anwendung bei typischen Symptomen funktioneller Verdauungsstörungen bestätigen.

\section{Schlüsselwörter}

Kümmelöl, Carvi aetheroleum, Pharmakologie, gastrointestinale Wirkungen, klinische Anwendung
Kümmelöl? - Auf einen ersten kurzen Blick in die aktuellen Monografien des „Hager“, der ESCOP und in den Assessment Report des HMPC gibt es scheinbar nur wenige Daten. Beruht die Anwendung denn überwiegend nur auf einer langjährigen Tradition und damit lediglich auf einer Plausibilität? Nein, keineswegs, wie dieser Beitrag zeigen soll. Wenn wir uns die bis heute vorliegenden Daten aus Pharmakologie und Klinik genauer anschauen, ergibt sich ein recht schlüssiges Bild.

Die aktuelle Monografie der ESCOP [5] und die des HMPC [2] benennen eine symptomatische Behandlung von Verdauungsbeschwerden wie Blähungen und Flatulenz sowohl bei oraler als auch bei kutaner Anwendung (Einreibung im Bereich des Abdomens). Die ESCOP nennt darüber hinaus auch die Anwendung bei flatulenzbedingten Koliken von Säuglingen und Kindern. In beiden Monografien werden diese Anwendungen als traditionell und damit als plausibel betrachtet.

Dies steht in schlüssiger Folge der Empfehlungen zu den Anwendungen des Kümmelöls in einigen relevanten früheren Werken (ohne Anspruch auf Vollständigkeit): So schrieb Leonhart Fuchs in seinem Kräuterbuch von 1543 zum „Wisenkümel“: „Der same von disem Kümel treibt den Harn / ist dem magen und dem mund ganz lieblich und dienstlich. Vertreibt die bläst und wind“ [10]. Gerhard Madaus [26] betrachtete die Früchte als auch das Öl als ein beliebtes Stomachikum und Karminativum, „welches bei Flatulenz, Meteorismus, Magenkrämpfen, Magenschwäche, Dyspepsie und Enteritis besonders Kindern gern verordnet wird“. In der Ausgabe von Hagers Handbuch der Pharmazeutischen Praxis von 1949 [9] wird das Öl als Stimulans und Karminativum bei Appetitlosigkeit, Magenkrampf und Flatulenz gelistet und die frühere Kommission E empfahl dann 1990 die innere Anwendung bei dyspeptischen Beschwerden wie leichte, krampfartige Beschwerden im MagenDarm-Bereich, Blähungen und Völlegefühl [22].

\section{Phytochemie}

Das ätherische Kümmelöl wird aus den trockenen Früchten von Carum carvi L. ( $\triangleright$ Abb. 1) durch Wasserdampfdestillation gewonnen. Das Öl besteht zu $50-65 \%$ aus (S)(+)-Carvon und zu 30-45\% aus (R)-(+)-Limonen [32], [14] ( Abb. 2). Ersteres ist für den typischen Geruch des Öls verantwortlich. Der Gehalt an Carvon im ätherischen Öl hängt vom Reifezustand der Kümmelfrüchte sowie von der Destillationszeit ab (die Zeit zur erschöpfenden Destillation ist für Carvon mit etwa 3,5 h deutlich kürzer als für Limonen mit etwa $8 \mathrm{~h}$ ). Weitere Bestandteile in geringeren Mengen (bis zu 1,5\%) sind zahlreiche weitere Terpene wie Myrcen, $\alpha$-Phellandren, $p$-Cymen und $\beta$-Caryophyllen sowie cis- und trans-Carveol, cis- und trans-Dihydrocarvon und trans-Dihydrocarveol [14]. 


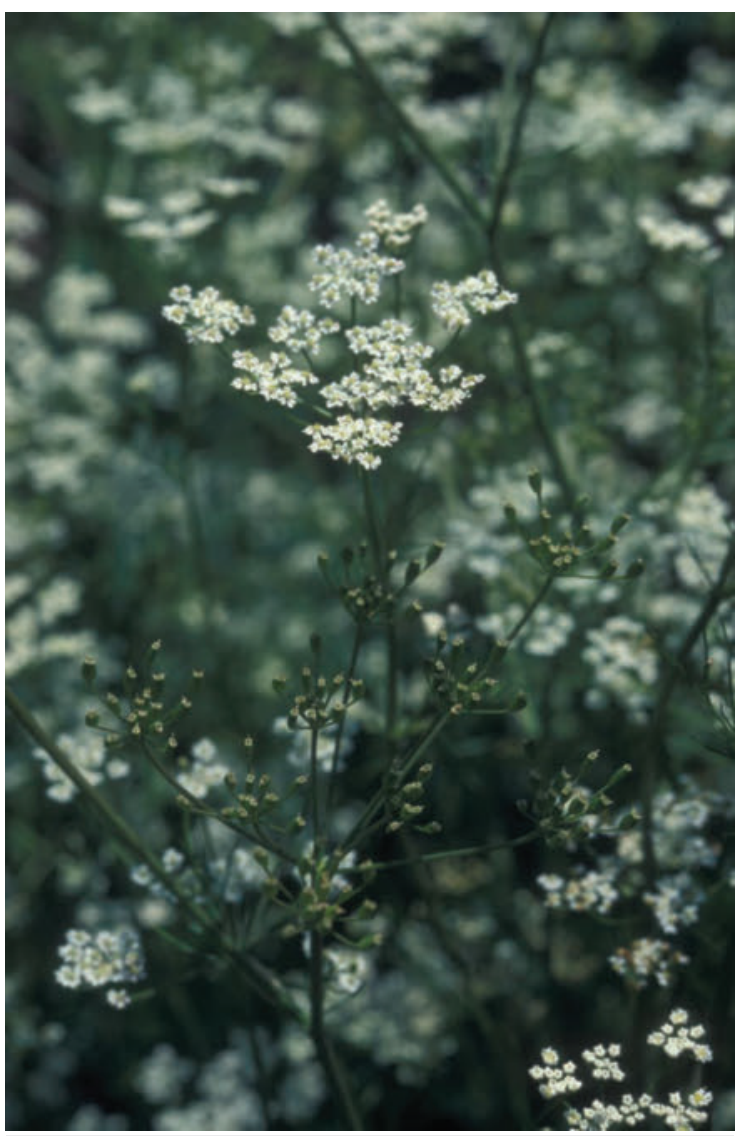

- Abb. 1 Der Echte Kümmel kann mehr als nur würzen er wirkt auch bei vielerlei Magen- und Darmproblemen. Die aromatischen Stoffe regen die Verdauung an, beseitigen Blähungen und regulieren die Darmflora. Quelle: Dr. Roland Spohn

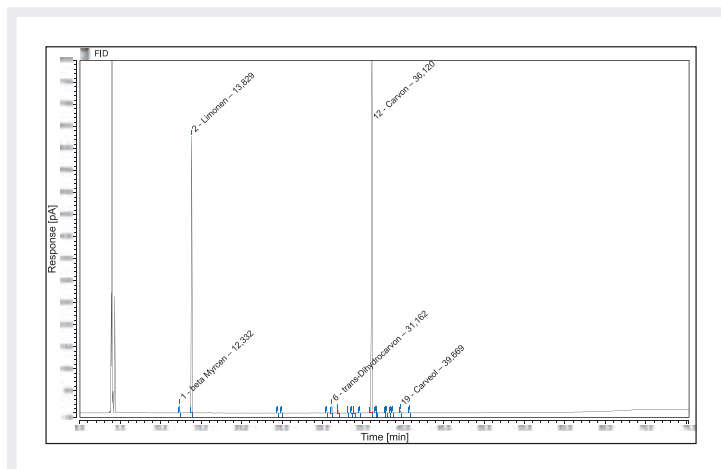

Abb. 2 Ein typisches Gaschromatogramm des Kümmelöls. Quelle: Dr. Willmar Schwabe

\section{Wirkungen}

Wenn auch bis dahin nur sehr wenige Daten zur experimentellen Pharmakologie des Kümmelöls bzw. der Droge Kümmelfrüchte vorlagen, postulierte Prof. Schilcher bereits Mitte der 1980er-Jahre folgende 5
Wirkmechanismen des Kümmels bzw. des ätherischen Öls [36], [37]:

- Lokale Stimulation der Magenschleimhaut, was den Vagusnerv aktiviere und zu einem Anstieg des Magentonus und der rhythmischen Kontraktion (Motilität) führe; das Ergebnis sei dann ein Entweichen der Luft aus dem Magen

- Anregung der direkten und reflektorischen Beeinflussung der Magensaftsekretion

- spasmolytische Wirkungen auf die glatte (Darm-) Muskulatur

- antiseptische Wirkung insbesondere auf Gärungsbakterien

- eine cholagoge Wirkung und damit eine verbesserte Verdauung (im Sinne einer Emulgierung) von Nahrungslipiden.

Dies als Postulat aus früherer Zeit vorangestellt, sind mittlerweile folgende Wirkungen - die alle direkt oder indirekt für die Anwendung bei Blähungen, Völlegefühl, Krämpfen und damit im Zusammenhang stehenden Schmerzen bedeutsam sind - für das Kümmelöl tatsächlich belegt:

\section{Spasmolytische und antispasmogene Wirkungen}

Publizierte Daten liegen vor zu antispasmodischen Wirkungen (Vorkontraktion durch Acetylcholin, Histamin, Carbachol sowie durch elektrische Spannung) und zu spasmolytischen Wirkungen an Trachea- bzw. Dünndarm-Segmenten des Meerschweinchens für das Kümmelöl [1], [8], [33] und für Extrakte aus den Kümmelfrüchten [7]. Auch wurde über antispasmogene (Acetylcholin, Kaliumchlorid) Eigenschaften am glattmuskulären Uterus der Ratte berichtet [35].

Neben diesen Eigenschaften des ätherischen Öls als Gesamtes sind diese auch für die einzelnen Terpene belegt: Für $(S)-(+)$-Carvon und $(R)-(+)$-Limonen eine leichte Relaxation des Meerschweinchen-Dünndarms [41] sowie antispasmogene Effekte bei durch Carbachol vorkontrahierten und antispasmogene und spasmolytische Effekte bei elektrisch stimulierten Längsmuskelpräparaten vom Ileum und Magenfundus der Maus [39].

\section{Antimikrobielle bzw. antibakterielle Wirkungen}

Es liegen viele Angaben zu antimikrobiellen bzw. antibakteriellen Eigenschaften des Kümmelöls bzw. von (+)-Carvon vor: bei Bacillus subtilis, Bacillus cereus, Pseudomonas aeruginosa, Candida albicans, E. coli, Saccharomyces cerevisiae, Aspergillus niger, Shigella dysenteriae, Vibrio cholerae, Staphylococcus aureus, Salmonella enteritidis, Micrococcus luteus, Proteus mirabilis, Pseudomonas tolaasii sowie bei Dermatophyten [3], [5], [6], [11], [14], [16], [38], [40], [44]. 
Beachtenswert ist ein möglicher selektiver Prozess: In vitro wurde das Wachstum der natürlichen Darmflora mit z. B. Lactobacillus spec. und Bifidobacterium spec. (erst) mit deutlich höheren MIC-Werten ( $\geq 2,2 \%$ im Vergleich zum Wachstum typischer potenziell pathogener Darmkeime wie Bacteroides fragilis, Candida albicans und Clostridium spp. (MIC-Werte hier 0,275-0,55\%) gehemmt [16].

Darüber ist nachvollziehbar, dass die karminative (entblähende) Wirkung des Kümmelöls auch auf antimikrobiellen Effekten durch verminderte intestinale Gärungsprozesse und damit Gasbildung durch pathogene Mikroorganismen beruhen kann.

\section{Gastrointestinale Motilität}

Die Auswirkungen von (R)-(-)- und (S)-(+)-Carvon auf die Darmmotilität wurden bei Mäusen durch Messung der Entleerung von Farbstoffen aus dem Magen und der Intensität der Darmtätigkeit bewertet. Im Vergleich zum Vehikel alleine war die Geschwindigkeit der Magenentleerung mit beiden Enantiomeren signifikant geringer und lag etwas über den Werten für die Kontrolle Loperamid. Beide verzögerten auch signifikant den Darmtransit, wenn eine flüssige Testmahlzeit direkt in den Zwölffingerdarm injiziert wurde, wobei die Auswirkungen mit denen der Positivkontrolle (Loperamid) vergleichbar sind. Zudem waren die spontanen und rhythmischen intragastrischen mittleren Amplituden der Druckwellen im Vergleich zu Vehikel alleine verringert, ohne die Stärke der Wirkung von Loperamid zu erreichen [39].

An humanen Dünn- und Dickdarmpräparaten aus chirurgischer Resektion wurden die Wirkungen von Kümmelund Pfefferminzöl auf die kontraktile (gemessen an zirkulären Glattmuskelstreifen; elektrische Stimulation kontraktiler Reaktionen) und sekretorische Aktivität (UssingKammer-Voltage Clamp-Technik) untersucht. Mit IC $\mathrm{I}_{50^{-}}$ Werten zwischen 7 und $127 \mu \mathrm{g} / \mathrm{ml}$ hemmte das Kümmelöl bei beiden Geweben konzentrationsabhängig die Muskelkontraktilität, ersichtlich an einer anhaltenden Muskelentspannung und einer Abnahme der phasischen Kontraktilität; die kontraktile Nervenreaktion wurde nicht beeinflusst. Die Hemmung der kontraktilen Aktivität, aber nicht die Muskelentspannung, wurde durch den L-Typ-Kalziumkanal-Aktivator Bay K8644 verhindert, nicht aber durch das Neurotoxin Tetrodotoxin (Hinweis auf Blockade von L-Typ-Kalziumkanälen). Gemessen wurde zudem eine Erhöhung der epithelialen Sekretion. Diese konnte durch Tetrodotoxin nicht blockiert werden, was auf eine direkte Aktivierung der Epithelzellen hinweist [23].

Die Wirkung einer intraduodenalen Verabreichung von Kümmelöl $(50 \mathrm{mg})$ auf die gastroduodenale Motilität wurde auch an gesunden Freiwilligen untersucht. Die
Ergebnisse zeigten eine signifikante Verringerung der Kontraktionsamplituden des Duodenums sowie eine Verkürzung der Kontraktionsdauer und der Amplituden im Magenkorpus und Antrum, was auf eine entspannende Wirkung des Öls auf die glatte Muskulatur hinweist [31].

\section{Physikochemische Eigenschaften}

Bereits vor vielen Jahren wurde über eine konzentrationsabhängige Reduktion der Schaumbildung durch Kümmelöl (und auch andere ätherische Öle) berichtet. Untersucht wurde dies in einer skalierten Glasröhre, in die künstlicher Magen- oder Darmsaft eingefüllt und mit Luft durchströmt wurde [15].

Mit einer etwas modifizierten Methode, bei der sich in der Glasröhre oberhalb der Flüssigkeit ein gleichmäßig steigender Schaum durch einen eingestellten Druck bildet, wurde dieses Experiment mit Kümmel- und Pfefferminzöl sowie deren Kombination wiederholt [21] ( $\triangleright$ Abb.3). Neben der Schaumhöhe wurde auch die Oberflächenspannung (Ringmethode nach Lecomte du Noüy mit Interfacial-Tensiometer) gemessen. Die Zugaben von Kümmel- als auch von Pfefferminzöl zu künstlichem Magen- oder Darmsaft führten zu einer deutlichen und konzentrationsabhängigen Abnahme der Schaumbildung ( $\mathrm{IC}_{50}$-Werte von 0,02-0,09\%) ( Abb.4). Die vorliegenden Untersuchungen demonstrieren, dass die ätherischen Öle im therapeutischen Dosisbereich über ausgeprägte schaumverhütende Effekte verfügen. Die beiden Öle allein, aber auch deren Kombination verringerten zudem konzentrationsabhängig die Oberflächenspannung mit maximal ca. $25 \%$.

Anzumerken ist, dass der hier gezeigte Effekt auf einer unmittelbaren Verfügbarkeit der ätherischen Öle im Experiment beruht; bei oraler Anwendung wird das Ausmaß einer entschäumenden Wirkung sicherlich beeinflusst sein durch den Prozess der Freisetzung und Verteilung im Darmlumen und ggf. auch von der Zusammensetzung der Darmflora.

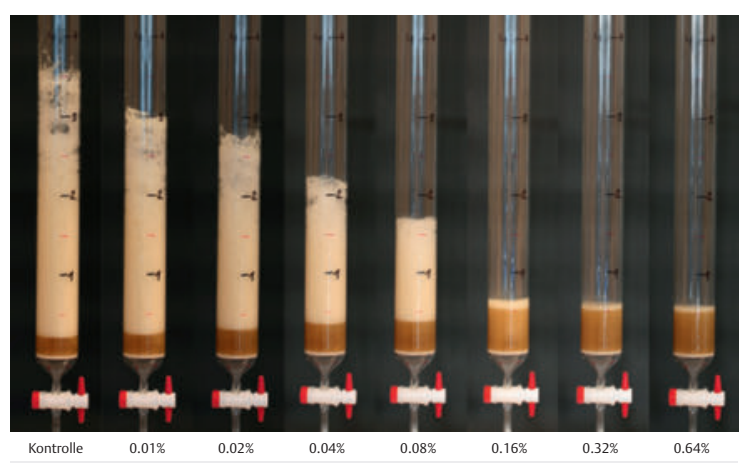

-Abb. 3 Hemmung der Schaumbildung in künstlichem Darmsaft durch ansteigende Konzentrationen einer Kombination von Pfefferminzöl und Kümmelöl (Mischungsverhältnis 9:5). Quelle: [21] 


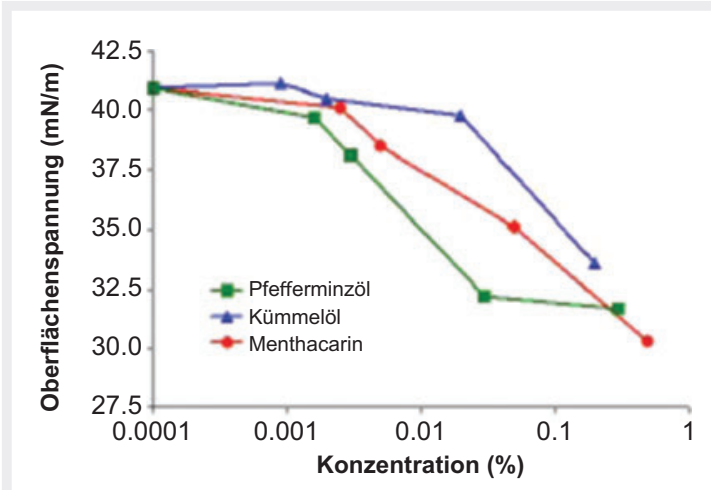

-Abb. 4 Dosisabhängige Verminderung der Oberflächenspannung durch Pfefferminz- und Kümmelöl und deren Kombination (Menthacarin ${ }^{\circledR}$ ) in künstlichem Darmsaft. Quelle: [21]

\section{Antinozizeptive Wirkungen}

Nachdem in einer In-vivo-Studie gezeigt wurde, dass ein analgetischer Effekt von (-)-Carvon in verschiedenen Modellen bei verminderter Nervenerregbarkeit vermindert ist [13] und dies auf einer Blockade von L-Typ-Kalziumkanälen beruht [42], s. auch [23], wurde gezeigt, dass die Nervenerregbarkeit verringert wird, indem spannungsgesteuerte Natriumkanäle auf reversible Weise blockiert werden. In diesen Experimenten (modifizierte GAP-Methode) erwies sich dann (+)-Carvon deutlich wirksamer als das Enantiomer (-)-Carvon [12].

Weiterhin wurden die Auswirkungen von (+)- und (-)-Carvon auf die glutamaterge spontane exzitatorische Übertragung in Neuronen der Substantia Gelatinosa adulter Ratten-Rückenmarkschnitte mithilfe der WholeCell-Patch-Clamp-Technik untersucht. Hier zeigte sich eine Aktivierung von TRPA1-Kanälen durch (+)-Carvon, ein Hinweis auf den Mechanismus der antinozizeptiven Wirkung des Kümmelöls [19]. TRPA1-Kanäle spielen nicht nur bei der Schmerzrezeption, sondern auch bei der gastrointestinalen Motilität eine Rolle; sie sind im Übrigen auch im Darm lokalisiert [45].

\section{Weitere Wirkungen}

In vivo zeigte sich eine kurative Wirkung des Kümmelöls an einer schnelleren Abheilung der durch Trinitrobenzolsulfonsäure (TBNS) induzierten Kolitis bei der Ratte [20]. Das $6 \mathrm{~h}$ nach der TBNS-Instillation (Kolon) oral applizierte Öl war wirksam im Dosisbereich von nur 100-400 $\mu \mathrm{l} / \mathrm{kg}$ Körpergewicht (ein wässrig-alkoholischer Extrakt aus den Früchten in Dosen von 100-400 mg/kg); das Ausmaß der Kolitis wurde mikroskopisch und histologisch bewertet, Positivkontrollen waren Prednisolon, Mesalazin und Hydrocortison.

\section{Daten aus klinischen Studien: Reizdarmsyndrom}

Eine der bekannten Anwendungsempfehlungen von Kümmelöl ist die Einreibung bei Säuglingen / Kleinkindern mit krampfartigen Magenschmerzen. Wenn auch nicht genau zu dieser Indikation in dieser Klientel, so liegen doch die Daten einer interessanten Studie vor, in der sich die „Kümmelölleibauflage nach Kneipp“ bei Patienten mit Reizdarmsyndrom als wirksam erwiesen hatte - und über die bereits in dieser Zeitschrift berichtet wurde [24], [25]:

In der monozentrischen, randomisierten, offenen Crossover-Studie wurden Patienten mit Reizdarmsyndrom behandelt. Alle erhielten in unterschiedlicher Reihenfolge alle 3 Interventionen für jeweils 30 min täglich über jeweils 3 Wochen: A) einen erwärmten Moor-Breiumschlag mit Kümmelöl (2\%ig in Olivenöl), B) eine erwärmte Leibauflage nur mit Olivenöl oder C) eine körperwarme Auflage mit Olivenöl. Hauptzielparameter war die Intensität der Reizdarmbeschwerden, erfasst mit der Irritable Bowel Syndrom Symptome Severity Scale, IBS-SSS; Nebenzielparameter waren u. a. Skalen zur Lebensqualität (EQ-5D, IBS-QOL). Das Ergebnis: Es wurde ein signifikanter Unterschied für den Symptomschweregrad zugunsten der Kümmelölgruppe im Vergleich zur Gruppe mit nur körperwarmem Olivenöl, nicht aber im Vergleich zum erwärmten Olivenöl festgestellt. Allerdings war die Responderrate (mind. 50 Punkte Symptomreduktion in der IBS-SSS) am höchsten für den Breiumschlag mit Kümmelöl (43,9\% vs. 20,0\% Gruppe B und 18,9\% für Gruppe C) sowie die Wertung einer angemessenen Wirkung mit 51,8\% in Gruppe A, vs. 23,5\% und 25,8 \% für Gruppen B und C.

Eine Anmerkung: Dass die topische Anwendung von Kümmelöl-neben Effekten der Wärme und der Massage-im Prinzip auch eine pharmakologische Wirkung ausüben könnte, ist durch den Nachweis der Resorption und Bioverfügbarkeit von auf dem unteren Abdomen appliziertem (S)-(+)-Carvon (in Erdnussöl) in einer früheren Studie gezeigt worden [18].

Möglicherweise reguliert das Kümmelöl in Richtung einer normalen Darmtätigkeit: Eine Studie aus dem Iran berichtete kürzlich über eine schnellere Wiederaufnahme der Darmmotilität (signifikant kürzere Zeit bis zum Hören der ersten Darmgeräusche bei der Auskultation, bis zum ersten Flatus, bis zum ersten Stuhlgang und kürzere Dauer des Krankenhausaufenthalts) nach operativen Eingriffen unter Vollnarkose (hier einem Kaiserschnitt). Die Patientinnen der Interventionsgruppe $(n=50)$ erhielten 6 und $7 \mathrm{~h}$ nach der Operation eine Dosis von jeweils $4 \mathrm{~g}$ Kümmel in einem Sirup (keine weiteren Angaben), die Kontrollgruppe $(n=48)$ erhielt einen Placebosirup [46]. 


\section{Heutige therapeutische Verwendung des Kümmelöls als Phytopharmakon}

Zubereitungen mit Kümmelfrüchten oder Kümmelöl als alleiniger Wirkstoff in Form von Fertigarzneimitteln sind nicht bekannt (das mag auch geschmacklich nicht jedermanns Sache sein). Es gab jedoch schon immer viele Kombinationsarzneimittel und auch Standardzulassungen mit Kümmelfrüchten oder dem Kümmelöl, so ist vor allem der „AFK“-Tee (Magenund Darm-Tee II mit Anis, Fenchel, Kümmel) neben den weiteren Magen- und Darm-Tees I-IX) eine sicherlich oft bewährte Standardzulassung. Basis dafür waren wohl die verschiedenen Kombinationsmonografien der Kommission E.

Eine moderne, in vielen Studien im RCT-Design geprüfte Arzneimittelspezialität enthält eine Kombination von $50 \mathrm{mg}$ Kümmel- und $90 \mathrm{mg}$ Pfefferminzöl in einer magensaftresistenten Weichkapsel (Carmenthin ${ }^{\circledR}$, in wiss. Publikationen Menthacarin $\left.{ }^{\circledR}\right)$. Für dieses Produkt liegt eine Reihe von Studien vor, in denen sich u. a. im Vergleich zu dem früher üblichen Cisaprid eine vergleichbare [27] und vs. Placebo eine sehr gute [29], [30], [34] Wirksamkeit zeigte, sogar auch bei der Langzeitanwendung von bis zu 12 Monaten [17], [43] bei nicht säurebedingter funktioneller Dyspepsie, in einer
Studie auch bei chronisch-entzündlichen Darmerkrankungen [4]. Eine jüngere Analyse der Daten aus 3 Studien (Metaanalyse von Subgruppen) ergab zudem eine Wirkung (Zielgröße: Schmerzempfinden) bei Dyspepsie-Patienten mit typischen Symptomen eines Reizdarms [28].

\section{Fazit}

Die in den 1980er-Jahren postulierten Wirkungen des Kümmelöls wurden mittlerweile in experimentellen und klinischen Studien vielfach gezeigt. Darüber hinaus gibt es Daten zu weiteren Wirkungen - das Potenzial des Kümmel(öls) ist offenbar immer noch nicht ganz erschöpfend geklärt und erschlossen.

\section{ABSTRACT}

Gastrointestinal effects of caraway oil, Carvi aetheroleum

The essential oil from Carum carvi L., Carvi aetheroleum, consisting mainly of the terpenes $(S)-(+)$-carvone and $(R)-(+)$-limonene, is used for digestive disorders accompanied by flatulence or even for flatulence-related colic. Predominantly, current experimental data gives indications or confirms the previously postulated effects. Worth mentioning are carminative (flatulence-inflating) effects 
in the gastrointestinal tract through spasmolytic or antispasmodic properties, a possible inhibition of intestinal fermentation processes through antimicrobial (limited to pathogenic germs) effects, a volume reduction through inhibition of the foaming of gastric and intestinal juices and thus a possible reduction of flatulence-related pain as well as direct antinociceptive properties. Results from clinical examinations and studies confirm the effect of caraway oil (alone or in combination with peppermint oil) in alleviating the typical symptoms of functional digestive disorders.

\section{Keywords}

Caraway oil, Carvi aetheroleum, pharmacology, gastrointestinal effects, clinical use

\section{Interessenkonflikt}

Der Autor erklärt, dass kein Interessenkonflikt besteht.

\section{Korrespondenzadresse}

Dr. Tankred Wegener
Consulting HMP
Brückstr. 11
69469 Weinheim
E-Mail: t.wegener@consulting-hmp.de

\section{Literatur}

[1] Brandt W. Spasmolytische Wirkung ätherischer Öle. Z Phytother 1988; 9: 33-39

[2] Committee on Herbal Medicinal Products (HMPC). European Union herbal monograph on Carum carvi L., aetheroleum, Final. EMA / HMPC / 715094 / 2013, 7 July 2015

[3] De M, De AK, Banerjee AB. Antimicrobial screening of some Indian spices. Phytother Res 1999; 13: 616-618

[4] Engel MA, Stracke B. Verbesserung der gesundheitsbezogenen Lebensqualität bei Patienten mit chronisch entzündlichen Darmerkrankungen nach einer 4-wöchigen Zusatztherapie mit Menthacarin. Z Phytother 2017; 38 (Suppl. 01): S20. doi:10.1055/s-0037-1607137

[5] European Scientific Cooperative on Phytotherapy (ESCOP). Monograph Carvi aetheroleum. Caraway Oil, Online Series, 2019

[6] Farag RS, Daw ZY, Abo-Raya SH. Influence of some spice essential oils on Aspergillus parasiticus growth and production of aflatoxins in a synthetic medium. J Food Sci 1989; 54: 7476

[7] Forster HB, Niklas H, Lutz S. Antispasmodic effects of some medicinal plants. Planta Med 1980; 40: 309-319

[8] Forster HB. Spasmolytische Wirkung pflanzlicher Carminativa. Tierexperimentelle Untersuchungen. Z Allg Med 1983; 59: 1327-1333

[9] Frerichs $G$ et al., Hrsg. Hagers Handbuch der Pharmazeutischen Praxis, 2. Berichtigter Neudruck, unveränderter Nachdruck 1949. Bd. 2. Berlin: Springer; 1949
[10] Fuchs L. Das Kräuterbuch von 1543, New Kreüterbuch (Nachdruck). Köln: Taschen Verlag, 2001

[11] Gniewosz M, Krasniewska K, Woreta M, Kosakowska O. Antimicrobial activity of a pullulan-caraway essential oil coating on reduction of food microorganisms and quality of baby carrot. J Food Sci 2013; 78: M1242-M1248

[12] Gonçalves JC, Alves Ade M, de Araújo AE et al. Distinct effects of carvone analogues on the isolated nerve of rats. Eur J Pharmacol 2010; 645: 108-112

[13] Gonçalves JC, Oliveira F de S, Benedito RB et al. Antinociceptive activity of (-)-carvone: evidence of association with decreased peripheral nerve excitability. Biol Pharm Bull 2008; 31: 1017-1020

[14] HagerROM 2018. Carum. In: Blaschek W, Hilgenfeldt U, Holzgrabe U, Moerike K, Reichling J, Ruth P, Hrsg. Hagers Enzyklopädie der Arzneistoffe und Drogen. Stuttgart: Wissenschaftl. Verlagsges.; 2018

[15] Harries N, James KC, Pugh WK. Antifoaming and carminative actions of volatile oils. J Clin Pharmacy 1978; 2: 171-177

[16] Hawrelak JA, Cattley T, Myers SP. Essential oils in the treatment of intestinal dysbiosis: A preliminary in vitro study. Altern Med Rev 2009; 14: 380-384

[17] Holtmann G], Stracke B. Sustained treatment effects of menthacarin and quality of life in patients with functional dyspepsia 8 weeks after the end of a 4-week placebo-controlled trial. United Eur Gastroenterol ] 2017; 5 (Suppl. 1): A790A791

[18] Jäger W, Mayer M, Reznicek G, Buchbauer G. Percutaneous absorption of the montoterperne carvone: implication of stereoselective metabolism on blood levels. J Pharm Pharmacol 2001; 53: 637-642

[19] Kang Q, Jiang C-Y, Fujita T, Kumamoto E. Spontaneous L-glutamate release enhancement in rat substantia gelatinosa neurons by $(-)$-carvone and $(+)$-carvone which activate different types of TRP channel. Biochem Biophys Res Comm 2015; 459: 498-503

[20] Keshavarz A, Minaiyan M, Ghannadi A, Mahzouni P. Effects of Carum carvi L. (caraway) extract and essential oil on TNBSinduced colitis in rats. Res in Pharm Sci 2013; 8: 1-8

[21] Koch E, Brauch S. Beitrag schaumhemmender Effekte von Pfefferminz- und Kümmelöl zu den karminativen Wirkungen von Menthacarin [Poster]. Z Phytother 2015; 36 (Suppl. 1): S34-S35. doi:10.1055/s-0035-1565971

[22] Kommission E. Monographie Carvi aetheroleum (Kümmelöl). BAnz Nr. 22a vom 01.02.1990

[23] Krueger D, Schäuffele S, Zeller F et al. Peppermint and caraway oils have muscle inhibitory and pro-secretory activity in the human intestine in vitro. Neurogastroenterol Motil 2020; 32: e13748

[24] Lauche R, Janzen A, Lüdtke R et al. Efficacy of caraway oil poultices in treating irritable bowel syndrome-A randomized controlled crossover trial. Digestion 2015; 92: 22-31

[25] Lauche R, Langhorst J. Studie zur Wirksamkeit von Kümmelölleibauflagen bei Patienten mit Reizdarmsyndrom. Z Phytother 2015; 36: 244-246

[26] Madaus G. Lehrbuch der biologischen Heilmittel, Bd. 4: Carum carvi. Leipzig: Georg Thieme; 1938 (Nachdruck mediamed Verlag, Ravensburg)

[27] Madisch A, Heydenreich C], Wieland V et al. Treatment of functional dyspepsia with a fixed peppermint oil and caraway oil combination preparation as compared to cisapride. A multicenter, reference-controlled double-blind equivalence study. ArzneimForsch / Drug Res 1999; 49: 925-932 
[28] Madisch A, Miehlke S, Labenz J et al. Effectiveness of Menthacarin on symptoms of irritable bowel syndrome. Wien Med Wochenschr 2019; 169: 149-155

[29] May B, Köhler S, Schneider B. Efficacy and tolerability of a fixed combination of peppermint oil and caraway oil in patients suffering from functional dyspepsia. Aliment Pharmacol Ther 2000; 14: 1671-1677

[30] May B, Kuntz HD, Kieser M, Köhler S. Efficacy of a fixed peppermint oil / caraway oil combination in non-ulcer dyspepsia. ArzneimForsch / Drug Res 1996; 46: 1149-1153

[31] Micklefield G, Jung O, Greving I, May B. Effects of intraduodenal application of peppermint oil (WS ${ }^{\circledR} 1340$ ) and caraway oil (WS ${ }^{\circledR} 1520$ ) on gastroduodenal motility in healthy volunteers. Phytother Res 2003; 17: 135-140

[32] Pharmacopoeia Europea, Europäisches Arzneibuch

[33] Reiter M, Brandt W. Relaxant effects on tracheal and ileal smooth muscles of the guinea pig. ArzneimForsch / Drug Res 1985; 35: 408-414

[34] Rich G, Shah A, Koloski N et al. A randomized placebocontrolled trial on the effects of Menthacarin, a proprietary peppermint- and caraway-oil-preparation, on symptoms and quality of life in patients with functional dyspepsia. Neurogastroenterol Motil 2017; 29. doi: $10.1111 / n m o .13132$

[35] Sadraei H, Ghannadi A, Takei-Bavani M. Effects of Zataria multiflora and Carum carvi essential oils and hydroalcoholic extracts of Passiflora incarnata, Berberis integerrima and Crocus sativus on rat isolated uterus. Int J Aromatherapy 2003; 13: $121-127$

[36] Schilcher H. Ätherische Öle-Wirkungen und Nebenwirkungen. Dtsch Apoth Ztg 1984; 124: 1433-1442

[37] Schilcher H. Pharmakologie und Toxikologie ätherischer Öle. Anwendungshinweise für die ärztliche Praxis. Therapiewoche 1986; 36: 1100-1112

[38] Seidler-Lozykowska K, Kedzia B, Karpinska E, Bocianowski J. Microbiological activity of caraway (Carum carvi L.) essential oil obtained from different origin. Acta Scientarium Agronomy 2013; 35: 495-500
[39] Silva CM, Wanderley CW, Lima-Junior FJ et al. Carvone $(\mathrm{R})-(-)$ and $(\mathrm{S})-(+)$ enantiomers inhibits upper gastrointestinal motility in mice. Flavour Fragr J 2015; 30: 439444

[40] Simic A, Rancic A, Sokovic MD et al. Essential oil composition of Cymbopogon winterianus and Carum carvi and their antimicrobial activities. Pharm Biol 2008; 46: 437-441

[41] de Sousa DP, Júnior GA, Andrade LN et al. Structure and spasmolytic activity relationships of monoterpene analogues found in many aromatic plants. Z Naturforsch C J Biosci 2008; 63: 808-812

[42] Souza FV, da Rocha MB, de Souza DP, Marçal RM. (-)-Carvone: Antispasmodic effect and mode of action. Fitoterapia 2013; 85: 20-24

[43] Storr M, Stracke B. Behandlungseffekt und Verträglichkeit von Menthacarin bei Patienten mit funktioneller Dyspepsie - ein 11-monatiges Follow-up. Z Gastroenterol 2017; 55: e220. doi:10.1055/s-0037-1605148

[44] Weseler A, Geiss HK, Saller R, Reichling J. A novel colorimetric broth microdilution method to determine the minimum inhibitory concentration (MIC) of antibiotics and essential oils against Helicobacter pylori. Pharmazie 2005; 60: 498-502

[45] Yang Y, Wang S, Kobayashi K et al. TRPA1-expressing lamina propria mesenchymal cells regulate colonic motility. JCI Insight 2019; 4(9).pii: 122402

[46] Yousefi SS, Sadeghpour O, Hamzehgardeshi Z, Sohrabvand F. The effects of Carum carvi (Bunium persicum Boiss) on early return of bowel motility after Caesarean section: doubleblind, randomized, placebo-controlled trial. J Family Reprod Health 2019; 13: 35-41

Bibliografie

DOI https://doi.org/10.1055/a-1102-1602 Zeitschrift für Phytotherapie 2020; 41: 55-61 (c) Georg Thieme Verlag KG Stuttgart · New York ISSN 0722-348X 